\title{
BMJ Open Comparison of Enteral versus Intravenous Potassium Supplementation in hypokalaemia in postcardiac surgery paediatric cardiac intensive care patients: prospective open label randomised control trial (EIPS)
}

\author{
Quratulain Merchant, ${ }^{1}$ Naveed ur Rehman Siddiqui, ${ }^{2}$ Amina Rehmat, ${ }^{3}$ \\ Muneer Amanullah, ${ }^{4}$ Anwar ul Haq, ${ }^{2}$ Babar Hasan ${ }^{2}$
}

To cite: Merchant Q, Rehman Siddiqui N ur, Rehmat $\mathrm{A}$, et al. Comparison of Enteral versus Intravenous Potassium Supplementation in hypokalaemia in postcardiac surgery paediatric cardiac intensive care patients: prospective open label randomised control trial (EIPS). BMJ Open 2014;4 e005124. doi:10.1136/ bmjopen-2014-005124

- Prepublication history for this paper is available online. To view these files please visit the journal online (http://dx.doi.org/10.1136/ bmjopen-2014-005124).

Received 25 February 2014 Revised 4 August 2014 Accepted 6 August 2014

\section{CrossMark}

${ }^{1}$ Medical College, Aga Khan University, Karachi, Pakistan ${ }^{2}$ Department of Pediatrics and Child Health, Aga Khan University, Karachi, Pakistan ${ }^{3}$ Department of Nursing,

Aga Khan University, Karachi, Pakistan

${ }^{4}$ Department of Surgery, Aga Khan University, Karachi, Pakistan

Correspondence to Dr Babar Hasan; babar.hasan@aku.edu

\section{ABSTRACT}

Background: Hypokalaemia is frequently encountered in the daily clinical practices of a paediatric cardiac intensive care unit (PCICU). It is a strong independent predictor of mortality in patients with heart failure. Thus, prompt potassium replacement therapy holds pivotal importance in therapy for hypokalaemia. Although intravenous potassium replacement (IVPR) in hypokalaemia is the preferred route in most intensive care settings, it is associated with known safety risks and can lead to arrhythmias, cardiac arrest and death if inappropriately administered. Enteral potassium replacement (EPR), with its superior safety profile, may be a better alternative to IVPR.

Outcome: Primary outcome To compare the efficacy EPR and IVPR for treatment of hypokalaemia. Secondary outcome measures include a comparison of adverse effects (hyperkalaemia, diarrhoea, gastrointestinal bleeds, nausea and vomiting) after EPR and IVPR and a comparison of the number of dose/s required to achieve resolution of hypokalaemia for each episode of hypokalaemia.

Methods and analysis: The Enteral Versus Intravenous Potassium Supplementation trial is designed as a randomised, controlled, non-blinded trial with two arms. Intervention arms will be block randomised on alternate weeks for IVPR and EPR. Recruited patients will receive treatment accordingly. For analysis, the percentage change in serum potassium levels in $\mathrm{mEq} / \mathrm{L}$ after each event of potassium replacement in both arms will be used as an end point to compare the efficacy EPR and IVPR for treatment of hypokalaemia.

Study setting: The study will be conducted at the PCICU at the Aga Khan University Hospital, Karachi. Ethics and dissemination: This study has been approved by the Ethics Review Committee and Clinical Trials Unit at The Aga Khan University with respect to scientific content and compliance with applicable research and human subjects regulations.
Strengths and limitations of this study

EIPS is the first prospective randomized control trial comparing the routes (enteral versus intravenous) for potassium replacement in the post cardiac-surgery pediatric patients in the PCICU.

- Previously, a retrospective review has shown comparable efficacy between the two routes.

- Research from this trial will lead the way for further research in this field, possibly bringing about a change in the management of hypokalemia in post-op patients and subsequently lower complications and morbidity associated with intravenous potassium replacement.

- EIPS is not a blinded study which may lead to a procedure bias. Blinding could not be carried out in this trial owing to different routes of administration of the same supplementation (enteral versus intravenous) and different time interval for checking serum levels in each arm (1 $\mathrm{h}$ after intravenous replacement and $2 \mathrm{~h}$ after enteral replacement).

- Confounding factors, such as concomitant use of diuretics and inotropic agents during the episode, have been identified and will be adjusted in the analysis.

Trial registration number: This trial is registered at Clinical Trials.Gov. Registration number: NCT02015962.

\section{INTRODUCTION}

Hypokalaemia is frequently encountered in the daily clinical practices of a paediatric cardiac intensive care unit (PCICU). Activation of the rennin-angiotensin-aldosterone system, the presence of an enhanced 
sympathetic tone and the use of potassium-wasting diuretics for a positive fluid balance increases the occurrence and consequences of severe hypokalaemia. ${ }^{1}$ Hypokalaemia is a strong independent predictor of mortality in patients with heart failure..$^{2-4}$ Thus, potassium replacement therapy is the cornerstone therapy for hypokalaemia. It is desirable to avoid hypokalaemia by the proactive style and aggressive replacement of potassium. There is mounting evidence that the serum potassium level should be maintained between 4.5 and5.5 mEq/L in acute cardiac injury settings. ${ }^{5} 6$

Although intravenous potassium replacement (IVPR) in hypokalaemia is the preferred route in most intensive care settings, it is associated with known safety risks. If inappropriately administered, IVPR can lead to arrhythmias, cardiac arrest and death. ${ }^{2} 78$ Given these risks, IVPR is considered a 'high-alert medication' by the Institute of Safe Medication practice. ${ }^{9}{ }^{10}$ Other issues with IVPR are the need for a central line that can lead to central line-related infection due to frequent access of the line during IVPR. Additionally, a larger volume of fluid is required during IVPR for delivery of the desired dose of potassium. This may not be preferred in postsurgical cardiac patients in whom a negative fluid balance is being achieved. Given all the aforementioned issues with IVPR, enteral potassium replacement (EPR), with its superior safety profile, may be a better alternative to IVPR. A retrospective review showed that the efficacy of EPR was comparable to that of IVPR in paediatric patients after congenital heart disease. ${ }^{8}$

We seek to explore this comparison between EPR and IVPR in a randomised prospective trial.

\section{Hypothesis}

Null hypothesis: There is no difference in the efficacy of IVPR and EPR during an episode of hypokalaemia.

Alternate Hypothesis: There is a mean difference of $15 \%$ in the change in serum potassium levels between the two groups, that is, IVPR and EPR during an episode of hypokalaemia.

\section{Outcomes}

Primary outcome: To compare the efficacy EPR and IVPR for treatment of hypokalaemia (measured as a change in serum potassium levels in $\mathrm{mEq} / \mathrm{L}$ after potassium replacement).

\section{Secondary outcomes}

1. Comparison of adverse effects after EPR and IVPR. Adverse effects that will be monitored are hyperkalaemia, diarrhoea, gastrointestinal (GI) bleeds, nausea and vomiting.

2. Comparison of the number of dose/s required to achieve resolution of hypokalaemia (as described per protocol) for each episode of hypokalaemia.

3. Efficacy EPR and IVPR for various degrees of severity of hypokalaemia, that is, mild, moderate or severe hypokalaemia. Efficacy for this analysis will be determined by the number of doses required for potassium supplementation to resolve an episode of hypokalaemia.

4. Efficacy of treatment based on the age of the patient. Efficacy for this analysis will be determined by the number of doses required for potassium supplementation to resolve an episode of hypokalaemia.

\section{Trial design}

The EIPS trial is designed as a randomised, controlled, non-blinded trial with two arms. The randomisation will be carried out as a block randomisation. Intervention arms will be block randomised on alternate weeks for IVPR and EPR (figure 1). Recruited patients will receive treatment as per the week of randomisation, that is, IVPR week versus EPR week.

Arm A (IVPR): Receiving intravenous potassium replacement to treat hypokalaemia.

Arm B (EPR): Receiving enteral potassium replacement to treat hypokalaemia

\section{METHODS}

\section{Definitions used for the study}

Normokalemia: Serum potassium level of $4.5-5.5 \mathrm{mEq} / \mathrm{L}$ is considered normal in patients with cardiovascular disease

Hyperkalaemia: Serum potassium $>5.5 \mathrm{mEq} / \mathrm{L}$.

Hypokalaemia: Hypokalaemia is defined as serum potassium $<4.4 \mathrm{mEq} / \mathrm{L}$

1. Symptomatic or asymptomatic: based on the presence of abnormal cardiac rhythm.

2. Severity: Mild, moderate or severe : based on the serum level of potassium.

- Mild: $3.5-4.4 \mathrm{mEq} / \mathrm{L}$

- Moderate: $2.5-3.4 \mathrm{mEq} / \mathrm{L}$

- Severe: $2.1-2.4 \mathrm{mEq} / \mathrm{L}$

Event and episode of hypokalaemia: Serum potassium $<4.4 \mathrm{mEq} / \mathrm{L}$ will be considered as hypokalaemia. This will mark the beginning of an EPISODE of hypokalaemia. Each potassium replacement will be considered an EVENT of hypokalaemia irrespective of whether

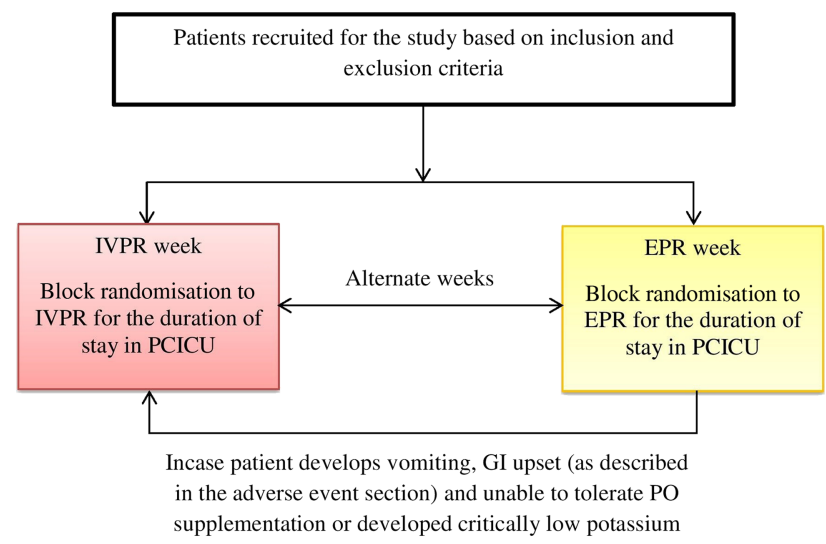

Figure 1 Block randomisation scheme for IVPR and EPR. 
hypokalaemia was completely resolved or not (ie, irrespective of whether the EPISODE of hypokalaemia as defined above ends or not). The EPISODE of hypokalaemia will end when the potassium level comes back to the normal range.

Efficacy of treatment: is defined as the change in potassium level (in $\mathrm{mEq} / \mathrm{L}$ ) after each IVPR or EPR, till specified otherwise.

\section{Study setting}

The study will be conducted at the PCICU at the Aga Khan University Hospital, Karachi. The PCICU has 150-160 postoperative admissions per year. Approximately $80 \%$ of the patients are under 5 years of age. The predominant $(\sim 70 \%)$ cardiac lesions are tetralogy of Fallot and ventricular septal defects. The mean length of PCICU stay is $\sim 2-3$ days. Clinically we anticipate 2-3 episodes, with 3-4 events in each episode of hypokalaemia in each patient during their PCICU stay.

\section{Eligibility criteria}

The following inclusion/exclusion criteria will be used for selection:

\section{Inclusion criteria}

- All patients undergoing surgical repair/palliation of congenital heart lesion at the Aga Khan University Hospital and admitted to the PCICU for postoperative management.

- Serum potassium levels $(<4.4 \mathrm{meq} / \mathrm{L})$ immediately postoperatively.

- Patients/parents willing to participate in this study.

- Have a central venous line for IVPR and an arterial line for monitoring and blood draws.

- Able to tolerate oral or nasogastric administration of medication for EPR.

- Age 1 month to 25 years.

\section{Exclusion criteria}

- Patients with acute renal failure (estimated clearance creatinine-ecCr $<50$ ).

- Patients with paralytic ileus, necrotising enterocolitis or GI bleeding.

- Patients with nausea, vomiting or diarrhoea prior to randomisation. Patients will not be excluded if these symptoms develop during the trial after initial recruitment. On the basis of the clinical decision, the route of potassium supplementation may be changed. Intention-to-treat analysis will be used for the primary end point.

- Patient with symptomatic hypokalaemia (manifested as a rhythm disturbance).

- Initial serum K level (post surgery) $<2.0 \mathrm{mEq}$.

\section{Consent procedure}

Informed consent will be taken by the principal investigator from each patient (or the parent in case of age $<13$ years) before going for cardiac surgery. The original signed consent form will be retained by the principal investigator, with a copy in the clinical notes and another provided to the participant.

Site-specific informed consent forms and assent forms (Urdu and English versions) have been approved by the Ethical Review Committee (ERC).

\section{Study recruitment}

Eligible patients will be recruited in the trial after being received in the PCICU postoperatively by the principal investigator. In case the child develops hypokalaemia, that is, serum potassium $<4.4$, the child will be followed till he/she reaches optimal potassium levels. This will be considered as a single episode. As long as the patient is in the PCICU, potassium monitoring will be continued as per the PCICU protocol and the patient will be recruited for the study in case he develops further episodes of hypokalaemia. Recruited patients will be enrolled in the intervention arms by the principal investigator and receive treatment accordingly. These patients will be part of the trial till their stay in the PCICU. The route of potassium replacement will be based on the week of therapy (block randomisation).

The recruited patients will be assigned a red or yellow protocol laminated sheet depending on the block (red for intravenous and yellow for enteral). In cases where the patient stays in the PCICU beyond the week and the block changes, the patient will keep following the route they were originally assigned to. EPR patients who develop side effects (eg, vomiting GI upset) with a subsequent need for parenteral potassium supplementation or who develop critically low levels of potassium $<2 \mathrm{mEq}$ (exclusion criteria) on enteral supplementation will be allowed to cross over and receive IVPR subsequently. An intention-to-treat analysis will be performed to account for cross-over patients.

\section{Study procedure and monitoring}

All postoperative patients in the PCICU are closely monitored using pulse oximetry, continuous electrocardiography, invasive or non-invasive blood pressures. Potassium supplementation as part of this trial will only be carried out in the PCICU under the aforementioned monitoring. As per the PCICU protocol, a $1 \mathrm{~mL}$ blood sample from an already placed art-line or central venous line is sent for analysis of serum potassium concentration in all the immediate postoperative patients. Potassium concentration will be assayed by means of auto analysers. The central lab will not be utilised to check the potassium level as the turnaround time for the lab values is $\sim 3-4 \mathrm{~h}$ in our institution. The point of care values are routinely used to treat hypokalaemia. Central lab values will be obtained on a daily basis or when a critically low or high value is seen on the auto analyser testing. Replacement of potassium based on the randomised group will be made either enterally (nasogastric tube or orally) or intravenously. A nasogastric feeding tube is regularly placed in our mechanically 
ventilated and sedated paediatric patients. These tubes were used to administer oral potassium supplementation in these patients. In the IVPR group, potassium will be given through a central line. As per a previously established protocol and bioavailability data, repeat serum potassium will be sent $1 \mathrm{~h}$ after replacement in the IVPR group and $2 \mathrm{~h}$ after replacement in the EPR group. Replacement and serum level monitoring will be performed till the episode of hypokalaemia is resolved. Further patient serum electrolyte monitoring will be determined by the patient's clinical status. No additional blood sample or monitoring will be performed as part of this trial and all such tests will be determined and dictated by the patient's clinical status, management plan and PCICU protocols.

An informal data safety monitoring board (DSMB) will be in place in the PCICU. The PCICU nurse manager and PCICU attending, not part of the trial, will be a part of this board. The PCICU nurse manager will be responsible for looking after the data collection and adverse effects during the trial period. The nurse manager will then report any adverse events to the clinical and the research team and the in-charge of the PCICU.

\section{Training}

Prior to the beginning of data collection, the nursing staff of the PCICU will be given a briefing session about the research. This session will include an overview of the research objectives and a review of the PCICU protocol to be followed. The nursing staff will be explained the block randomisation process for the research and reinforced about stringent monitoring of side effects.

All these trainings would be conducted prior to the study start-up and there will be a pre-recruitment trial period to make sure that the protocol practices are in place and the data collection runs smoothly.

\section{Study drugs, drug management}

We will use the following protocol established in our unit for potassium replacement:

Study drug management (procurement, randomisation, dispensing, log maintenance, drug accountability, drug returns) will be carried out by the institutional clinical trial unity/main pharmacy in accordance with the protocol.

\begin{tabular}{|c|c|}
\hline $\begin{array}{l}\text { Serum potassium } \\
\text { level (mEq) }\end{array}$ & $\begin{array}{l}\text { Potassium replacement } \\
\text { (intravenous and enteral) }\end{array}$ \\
\hline $4.0-4.4$ & $0.1 \mathrm{mEq} / \mathrm{kg} / \mathrm{dose}$ \\
\hline $3.5-3.9$ & $0.3 \mathrm{mEq} / \mathrm{kg} / \mathrm{dose}$ \\
\hline $3.0-3.4$ & $0.5 \mathrm{mEq} / \mathrm{kg} / \mathrm{dose}$ \\
\hline $2.5-2.9$ & $0.7 \mathrm{mEq} / \mathrm{kg} / \mathrm{dose}$ \\
\hline $2.1-2.4$ & $1 \mathrm{mEq} / \mathrm{kg} / \mathrm{dose}$ and call physician \\
\hline
\end{tabular}

Parental potassium chloride

'Potassium chloride $25 \mathrm{Meq} / 25 \mathrm{~mL}$ ' available in ampoule form. It comes under 'High Alert Medication'.

\section{Dosage}

The dose of IVPR depends on the serum potassium level (see table 1). The usual paediatric maximum dose of potassium is $3 \mathrm{Meq} / \mathrm{kg}$ /day or $40 \mathrm{Meq} / \mathrm{m}^{2} /$ day (for intravenous intermittent infusion in case of severe potassium depletion) and the patient should have continuous ECG monitoring.

\section{Route}

It can be administered either from the peripheral line or from the central line along with plenty of intravenous fluids.

\section{Dilution}

Potassium chloride must be diluted prior to parenteral administration; for dilution, the maximum recommended concentrations are as follows:

1. For peripheral line: $8 \mathrm{Meq} / 100 \mathrm{~mL}$ or $80 \mathrm{Meq} / \mathrm{L}$

2. For central line: $15 \mathrm{Meq} / 100 \mathrm{~mL}$ or $150 \mathrm{Meq} / \mathrm{L}$, for fluid restricted patient: $20 \mathrm{Meq} / 100 \mathrm{~mL}$ or $200 \mathrm{Meq} / \mathrm{L}$

$0.9 \%$ sodium chloride, $5 \%$ dextrose water, $10 \%$ dextrose water, $1 / 2$ strength saline, $1 / 5$ saline, $1 / 3$ saline are compatible diluents for potassium chloride.

\section{Infusion rate}

For peripheral line: (maximum infusion rate)

- In case of mild to moderate hypokalaemia, IVPR must be infused at the rate of $10 \mathrm{Meq} / \mathrm{h}$.

- In case of severe hypokalaemia, IVPR must be infused at the rate of $40 \mathrm{Meq} / \mathrm{h}$

For central line: (maximum infusion rate)

- $15 \mathrm{Meq}$ in 1-2 $\mathrm{h}$ and in fluid restricted patient $20 \mathrm{Meq}$ in $1-2 \mathrm{~h}$.

\section{Storage and stability}

Store at room temperature after dilution (either diluted at patient's bed site or in sterile area). It can remain stable for $24 \mathrm{~h}$ at room temperature. Use only clear solution.

\section{Oral potassium chloride}

It is available in syrup form with a concentration of $13.33 \mathrm{Meq} / 5 \mathrm{~mL}$. It can be administered with plenty of fluids and/or food to reduce the stomach discomfort and irritation. It can be mixed with orange juice to mask its unpleasant taste.

\section{Dosage}

Maximum dose in $24 \mathrm{~h}: 240 \mathrm{mEq}$, divided into 2-3 doses. Maximum per dose: $60 \mathrm{mEq}$ (see table 1).

\section{Stability}

Store at room temperature. 


\section{Side effects}

Diarrhoea, nausea, vomiting, stomach pain, flatulence, bradycardia, arrhythmia, chest pain, mental confusion, local tissue necrosis with extravasation, pain at the site of injection, dyspnoea, phlebitis.

\section{Contraindication}

Severe renal impairment, untreated Addison's disease, hyperkalaemia, severe tissue trauma.

\section{Drug interaction}

It can increase the effects of ACE inhibitors, potassium sparing diuretics and salt substitutes when given concomitantly with potassium chloride. ${ }^{11}$

\section{Adverse events}

Adverse effects of potassium supplementation that will be monitored are hyperkalaemia (defined as potassium levels $>5 \mathrm{mEq} / \mathrm{L}$, arrhythmias (defined as any rhythm other than sinus during or within $2 \mathrm{~h}$ of potassium replacement), diarrhoea (described as $>2$ loose stools or a single watery stool during or within $2 \mathrm{~h}$ of potassium replacement, GI bleeds, nausea and vomiting (during or within $2 \mathrm{~h}$ of potassium replacement).

The adverse events will be monitored on an hourly basis by the PCICU nursing staff as per the PCICU protocol. These adverse events will be documented on the PCICU nursing sheet (as per routine) and the data gathered every morning by the principal investigator or one of the coinvestigators. The on-call physician will be contacted, as per routine, by the nursing staff to determine any change in the mode/dose of potassium replacement if adverse events occur. The on-call physicians will in turn contact either the principal investigator or one of the coinvestigators to further discuss the plan.

Adverse events such as diarrhoea, GI bleeds, nausea and vomiting will be reported to the institution's ERC within 7 days of their coming to the PI's knowledge.

\section{Sample size calculation}

Using a power of $90 \%$, $p$ value of $<0.05 \%$ (95\% for onesided CI and $90 \%$ for two-sided CI) a total of 310 events of hypokalaemia are required to reject the null hypothesis.

\section{Statistical analysis}

The primary outcome of the study is to compare the efficacy EPR and IVPR for treatment of hypokalaemia. The end points that will be used to judge this are as follow:

- Percentage change in serum potassium levels in $\mathrm{mEq} / \mathrm{L}$ after each event of potassium replacement by both the methods, calculated as:

Post supplementation levels-presupplementation levels/ presupplementation levels.

- Number of potassium supplementations needed to treat the episode of hypokalaemia in each arm (EPR and IVPR)
- The time needed to treat an episode of hypokalaemia completely in each arm (EPR and IVPR)

The secondary outcomes of this study are to assess the number of adverse events in each arm (EPR and IVPR).

Data will be entered in SPSS and analysis will be performed for each hypokalaemic event and episode. For each episode, an intention-to-treat analysis will be performed. As the sample size is $>30$, using the center limit theorem, parametric tests will be used. Student $\mathrm{t}$ test will be used for analysis of continuous variables, while $\chi^{2}$ will be used for categorical variables.

Diuretic dosing is a component of the data collection and dosage of diuretics in $\mathrm{mg} / \mathrm{kg} / \mathrm{h}$ will be calculated for each episode. The process of block randomisation should help in an equal distribution between the two groups. At the analysis level, a multivariate linear regression will be performed to account for confounding variables like diuretic or inotropic use.

Patient demographic variables will be analszed only once on a patient-to-patient basis. For potassium level analysis, cumulative data from events and episodes of hypokalaemia will be analysed.

Participants who deviate from the intervention protocols would be excluded from the final analysis.

An interim analysis of the data will be carried out midway during the study, that is, after 155 events. The purpose of this interim analysis will be to assure protocol compliance, accurate completion of record forms and to monitor the adverse effects.

\section{Timeline}

After ERC approval, recruitment will be carried out and data collected. The expected time for completing this study will be $\sim 6$ months and a manuscript will be prepared within 6 months of completion of the study.

While in the PCICU in the postoperative period, the potassium will be monitored as per the ICU protocol. In case the child develops hypokalaemia, that is, serum potassium $<4.5$, the child will be included as a part of the study and will be followed till he reaches optimal potassium levels. There is no long-term follow-up once the patient is discharged from the PCICU.

\section{Data collection, storage and record keeping}

The data abstraction form will be used to abstract patient data for the study.

Data will be collected by a single principal investigator throughout the duration of the study to prevent any reporter's bias.

The data will be kept safe under lock at all times and will be simultaneously added to a password protected SPSS file. The other investigators will also be crosschecking the data.

\section{Discontinuation rules}

Regardless of the study, during the past 3 years (20102012), mortality in the PCICU remains 0-3 patients/ month out of the 10-30 patients admitted each month. 
The average of the percentages of monthly mortality in the past three years $(2010: 7.8 \% ; 2011: 5.2 \% ; 2012: 8.2 \%)$ is $7.1 \%$. If the mortality during the study interval exceeds this threshold, it will not be attributed to chance and further investigation of the research methodology will be warranted.

Contributors $\mathrm{BH}, \mathrm{QM}, \mathrm{AH}, \mathrm{MA}$ and NRS contributed to the development of the protocol and wrote the protocol. QM, BH, AR and NRS have written and revised the manuscript for submission. MA, AR and $A H$ were part of the team that conceived and planned the trial. All authors have read and approved the final manuscript.

Competing interests None.

Ethics approval This study has been approved by the ERC and Clinical Trials Unit at The Aga Khan University with respect to scientific content and compliance with applicable research and human subjects regulations. The protocol, site-specific informed consent forms (Urdu and English versions), participant education and recruitment materials, and other requested documents—and any subsequent modifications-also will be reviewed and approved by the ethical review bodies (ERC).

Provenance and peer review Not commissioned; externally peer reviewed.

Open Access This is an Open Access article distributed in accordance with the Creative Commons Attribution Non Commercial (CC BY-NC 3.0) license, which permits others to distribute, remix, adapt, build upon this work noncommercially, and license their derivative works on different terms, provided the original work is properly cited and the use is non-commercial. See: http:// creativecommons.org/licenses/by-nc/3.0/

\section{REFERENCES}

1. Schaber DE, Uden DL, Stone FM, et al. Intravenous $\mathrm{KCl}$ supplementation in pediatric cardiac surgical patients. Pediatr Cardiol 1985;6:25-8.

2. Schulman M, Narins RG. Hypokalemia and cardiovascular disease. Am J Cardiol 1990;65:4E-9E; discussion 22E-23E

3. Gennari FJ. Hypokalemia. N Engl J Med 1998;339:451-8.

4. Podrid PJ. Potassium and ventricular arrhythmias. Am J Cardiol 1990;65:33E-44E; discussion 52E

5. Leier CV, Dei Cas L, Metra M. Clinical relevance and management of the major electrolyte abnormalities in congestive heart failure: hyponatremia, hypokalemia, and hypomagnesemia. Am Heart $J$ 1994;128:564-74.

6. Macdonald JE, Struthers AD. What is the optimal serum potassium level in cardiovascular patients? J Am Coll Cardiol 2004;43:155-61.

7. Schaber DE, Uden DL, Stone FM, et al. Intravenous $\mathrm{KCl}$ supplementation in pediatric cardiac surgical patients. Pediatr Cardiol 1985;6:25-8.

8. Moffett BS, McDade E, Rossano JW, et al. Enteral potassium supplementation in a pediatric cardiac intensive care unit: evaluation of a practice change. Pediatr Crit Care Med 2011;12:552-4.

9. Institute for Safe Medication Practices. http://www.ismp.org/ Newsletters/acutecare/articles/20071004.asp (accessed 2 Aug 2009).

10. Institute for Safe Medication Practices. http://www.ismp.org/Tools/ highalertmedications.pdf (accessed 2 Aug 2009).

11. Lacy CF, Armstrong LL, Goldman MP. 'Lexi-Comp's drug information handbook. 13 edn. Hudson: Lexi Comp. 2006:1225-6. 